\title{
Microelectronic Fabrication of Transition Edge Sensors
}

\author{
K. L. Nelms, S. Ali, A. Dosaj, D. Liu, D. McCammon, L. E. Rocks, W. T. Sanders, P. Timbie, \\ J. E. Vaillancourt \\ University of Wisconsin-Madison, Department of Physics, 1150 University Ave., Madison, WI, \\ 53706-1390
}

Achieving optimum spectral energy resolution in conventional scanning electron microscopes (SEM) can be accomplished by using microcalorimeters. Improvements in device design are being studied, although many research groups have developed fabrication techniques that produce consistent results [1]. Here, we discuss the fabrication method that we are using to produce microcalorimeters using superconducting transition edge sensors (TES) as thermometers, and some of the roadblocks we are observing.

Many materials have been employed to engineer TES devices. We begin by depositing a $1 \mu \mathrm{m}$ low stress silicon nitride layer onto a $<100>$ silicon wafer. A molybdenum $(\mathrm{Mo}) / \operatorname{copper}(\mathrm{Cu})$ bilayer is then deposited via ebeam evaporation without breaking vacuum between depositing the layers. The Mo, $\sim 400 \AA$ in thickness, is evaporated at an elevated substrate temperature of $\sim 565$ ${ }^{\circ} \mathrm{C}$ to facilitate optimum grain size and shape. The $\mathrm{Cu}$ layer, $\sim 1740 \AA$ in thickness, is then evaporated at a much cooler substrate temperature of $\sim 100{ }^{\circ} \mathrm{C}$.

To obtain our goal of producing a $400 \mu \mathrm{m}$ x $400 \mu \mathrm{m}$ TES pad with Mo leads, the bilayer is fabricated via various masking steps involving both wet and dry etches. The general microprocessing consists of the following steps:

1. Wet etch two trenches in the $\mathrm{Cu}$ to gain access to the Mo layer. These trenches define the sides of the TES pad.

2. Using the remaining $\mathrm{Cu}$ as a mask, the exposed Mo is etched via dry plasma. This process laterally etches the Mo resulting in the $\mathrm{Cu}$ overhanging the Mo layer by $\sim 300 \mathrm{~nm}$. The $\mathrm{Cu}$ overhang is fabricated to eliminate superconducting shorts along the edges of the device.

3. Wet etch the $\mathrm{Cu}$ to define the ends of the TES pad.

4. Wet etch the Mo to define the leads.

5. The device, to be a good thermometer, must be thermally isolated. We achieve this by etching a window in the silicon substrate. $\mathrm{KOH}$ is used to etch the silicon from the back of the wafer while the device is covered for protection. The $\mathrm{KOH}$ etch will follow the (111) planes in the silicon and stop on the silicon nitride layer.

The primary problem present in our fabrication method revolves around $\mathrm{Cu}$ technology. To process the $\mathrm{Cu}$, we have chosen ammonium persulfate as a wet etchant. After the $\mathrm{Cu}$ is etched away from the underlying Mo, we have consistently measured a $\mathrm{Cu}$ undercut of $\sim 3 \mu \mathrm{m}$. However, when etching a $\mathrm{Cu}$ layer that is not part of a Mo/Cu bilayer, there is no visible undercut. 
To treat the $\mathrm{Cu}$ layer and attempt to eliminate undercutting, the bilayer was placed in a $1 \%$ Benzotriazole (BTAH) solution immediately after the evaporation process. BTAH, a known $\mathrm{Cu}$ corrosion inhibitor, showed no proof of stopping the undercutting problem. However, we are currently testing whether the chemical shows proof of hindering $\mathrm{Cu}$ oxidation.

To further assess the undercutting concern, we are investigating the root cause. One possibility is that the percentage of exposed Mo presents an accelerated $\mathrm{Cu}$ undercut. Tests such as using a highly diluted etchant, cooling the etchant and etching small areas of copper are being conducted. Once the origin of the undercutting is determined, a new mask set will be designed to compensate for the problem.

[1] P.Tan et al., Low Temperature detectors, (2002) AIP Conference Proceedings No. 605, and references therin.

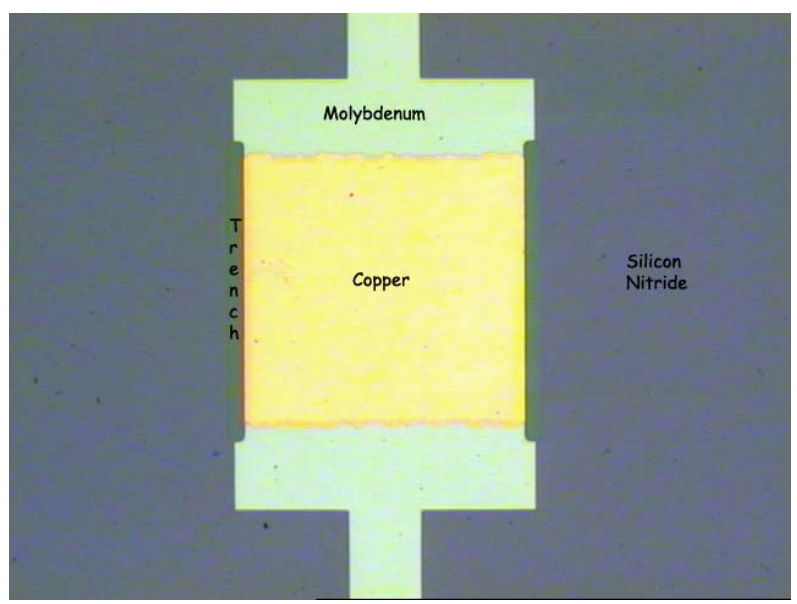

Figure 1: This light microscopy image shows a 400 x $400 \mu \mathrm{m}$ TES device with Mo leads. The trenches described in step one are visible along the vertical sides of the pad.

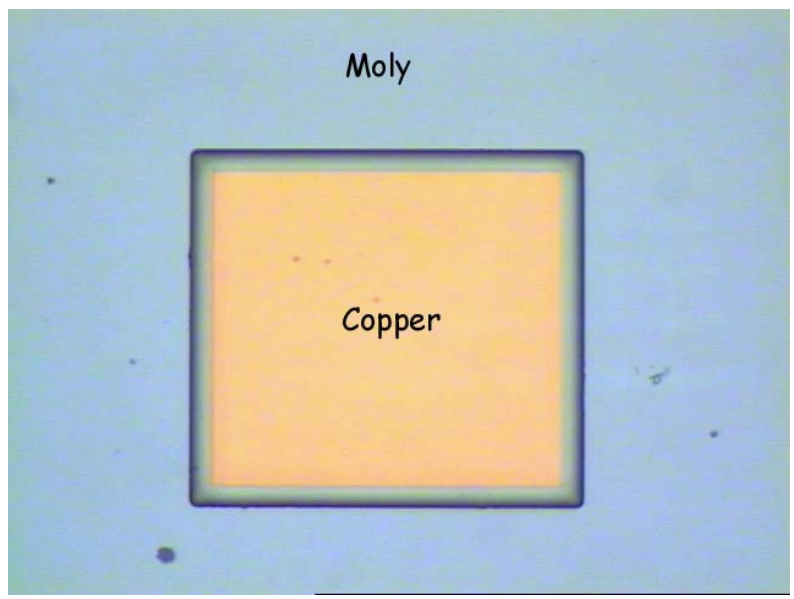

Figure 2: This light microscopy image shows a $200 \times 200 \mu \mathrm{m} \mathrm{Cu}$ pad with the photo-resist (PR) still covering the $\mathrm{Cu}$ layer. The dark line surrounding the $\mathrm{Cu}$ layer defines the edge of the transparent PR layer. The undercutting of the $\mathrm{Cu}$ layer is represented by the space between the $\mathrm{Cu}$ edge and the edge of the photo-resist. 